\title{
HISTOPATHOLOGICAL STUDY OF THE PROTECTIVE EFFECT OF SPIRULINA PLATENSIS ON FETAL LIVER OF MICE WITH ACUTE FATTY LIVER OF PREGNANCY
}

\author{
MARYAM KARGARZADEH ${ }^{1 *}$, MITRA HEIDARI NASRABADI ${ }^{1 *}$, AREZO DASTPAK $^{1}$, HANIEH KARGARZADEH ${ }^{2}$
}

${ }^{1}$ Department of Biology, Faculty of Basic Sciences, Central Tehran Branch, Islamic Azad University, Tehran, Iran. ${ }^{2}$ Center of Molecular and Macromolecular Studies, Polish Academic of Sciences, Sienkiewicza 112, 90-363, Łódz, Poland.

Email: maryam3497@gmail.com

Received: 17 May 2020, Revised and Accepted: 29 June 2020

ABSTRACT

Objective: The protective effect of Spirulina platensis on the fetal liver of mice with acute fatty liver of pregnancy was investigated.

Methods: Small female mice were divided into four groups: A control group with a standard diet, a high-fat diet to induce liver steatosis, a high-fat diet plus Spirulina, and a high-fat diet plus Simvastatin given through gavage to protect the liver against steatosis. After 2 months, the female mice became pregnant by polygamy method at the same time they were treated by different diets. On day 17 , the fetuses were removed by $\mathrm{C}$-section, and histological studies were carried out on their livers.

Results: The results showed a significant decrease in liver steatosis in the group treated by Spirulina compared with the other groups (p<0.05). The fatty liver of pregnancy could lead to liver failure and death in both mother and fetus, and medications like Simvastatin that is used for the treatment of fatty liver are harmful to the fetus. However, Spirulina shows a positive effect on the treatment of both fetus and mother.

Conclusions: The results of this study show that Spirulina is an effective medical supplement in the treatment of fatty liver of pregnancy.

Keywords: Acute fatty liver of pregnancy, high-fat diet, Spirulina, liver steatosis, fatty liver of pregnancy, fetal liver.

(C) 2020 The Authors. Published by Innovare Academic Sciences Pvt Ltd. This is an open access article under the CC BY license (http://creativecommons. org/licenses/by/4. 0/) DOI: http://dx.doi.org/10.22159/ajpcr.2020.v13i8.38355

\section{INTRODUCTION}

The liver is one of the most important organs in the body with an important role in detoxification, storage of glycogen, and metabolism of fats and carbohydrates. One of the most important and common diseases of the liver are the fatty liver which is divided into three types: Alcoholic fatty liver, non-alcoholic fatty liver, and fatty liver of pregnancy. What makes this condition worse is the irreversible destruction of liver cells if the disease is not diagnosed in the early stages. The fatty change could be subdivided into microvesicular and macrovesicular steatosis. In macrovesicular steatosis, the hepatocytes contain one bulky vacuole that displaces the nucleus. This type is mostly caused by obesity, diabetes, or alcohol abuse. In microvesicular steatosis, the cytoplasm is occupied by several small fat vesicles, and the nucleus remains centrally placed. It usually results from mitochondrial $\beta$-oxidation defects, which are usually associated with toxins or metabolic disorders [1].

Acute fatty liver of pregnancy (AFLP) is a rare but important disease that occurs in the third trimester of pregnancy. AFLP is one of the factors causing the microvesicular fatty liver. From the histological point of view, in microvesicular fatty liver, cell necrosis is minor and variable; the hepatocytes become swollen and have central nuclei with prominent nucleoli. In some examinations, centrizonal cholestasis is seen, and examinations by electron microscopes show that the mitochondria are swollen and pleomorphic, which would also decrease the oxidation of fatty acids, and the ribosomal patterns which are on the rough endoplasmic reticulum are seen abnormal [2]. Abdominal pain, nausea, vomiting, and fatigue are common symptoms of AFLP. Sometimes the mothers have been reported to experience hypoglycemia, hepatic encephalopathy, and moderate to severe coagulopathy, and preeclampsia [3]. Laboratory experiments show an increase in bilirubin and aminotransferase levels, and sometimes it involves neutrophilic leukocytosis, and with the progression of the disease, thrombocytopenia and hypoalbuminemia may also be seen $[3,4]$. In diagnosing this disease, examining the white blood cells shows a decrease in platelets and normoblasts [5]. Disseminated intravascular coagulation is also common in this disease [6]. Fibrinogen, thromboplastin, and prothrombin are usually abnormal in this disease, and the blood uric acid level increases. As the disease has drastic effects on both mother and child, diagnosis must be made early on. AFLP diagnosis is usually made based on laboratory findings and imaging appearances. In general, if AFLP is not diagnosed early on, it will cause significant maternal and fetal mortality. The main cause of the disease remains unknown; however, molecular studies show that the leading cause of AFLP is mitochondrial dysfunction [7-9]. AFLP usually occurs after unsuccessful or successive pregnancies for the mother. Pathophysiological studies of AFLP show defects in mitochondrial fatty acid beta-oxidation. In general, in normal conditions, no abnormal fatty oxidation is seen in a person who is heterozygous for enzymatic mutations in fatty acid oxidation. However, when a heterozygous mother carries a fetus that is homozygous for such mutations, a lot of fetal fatty acids return to the mother's circulation. This extra burden of long-chain fatty acids, and accumulation of triglyceride causes hepatic fat deposition and impaired hepatic function [3]. Natarajan et al. [10] showed that one of the key factors causing liver diseases is oxidative stress, and, in fact, microvesicular liver steatosis leads to mitochondrial dysfunction and oxidative stress. Oxidative stress was also found in preeclampsia patients, a condition which is found in $40 \%$ of the patients with AFLP. It is well-known that the most important cellular source of free radicals in preeclampsia patients is mitochondria; additionally, peroxisomes play a role in fatty acids oxidation [11]

Most patients, especially pregnant women, cannot bear the adverse effects of chemical medicines. However, biological substances, which are derived from plants and constitute an important part of modern pharmacotherapy, have few side effects on the patients. Some medications that are used for the treatment of chronic liver diseases are probably used during pregnancy, too; however, some medications 
are toxic for the fetus and therefore are not prescribed. As maternal nutrition is an important factor during pregnancy, proper nutrition could help the fetus develop well and ensures the health of the fetus; however, incorrect or unbalanced maternal nutrition causes fetal disorders [12].

Spirulina is a blue-green alga from the Oscillatoriaceae family, which is typically found in tropical and subtropical areas. The nutritional value of Spirulina is high. Spirulina contains a great amount of protein (60-70\% of its dry weight), a lot of anti-oxidants such as $\beta$-carotene, phycocyanin, and elements such as $\mathrm{K}, \mathrm{Na}, \mathrm{Ca}, \mathrm{Mg}, \mathrm{Fe}, \mathrm{Zn}$, vitamins (tocopherols), essential amino acids, and polyunsaturated fatty acids, especially $\gamma$-linolenic acid, phenolic compounds amino acids, gamma-Linolenic acid, and minerals [13-15]. Thanks to being rich in nutrients, and having therapeutic effects and non-toxicity, Spirulina has been known as one of the most important dietary supplements and useful in the prevention of some diseases in the $21^{\text {st }}$ century $[16,17]$. Consuming Spirulina supplements are suggested for the prevention and control of hypocholesterolemia [18], hyperglycerolemia [19], galactosamine-induced hepatotoxicity [20] obesity, inflammation [21], cancer [22], cardiovascular diseases [23], insulin resistance, diabetes, nonalcoholic fatty liver, malnutrition, anemia, allergic rhinitis, toxicity of substances [14,16,19], and also for its analgesic effect [24]. Due to its antioxidant properties, Spirulina Platensis has drawn a lot of attention for removing hydroxyl radicals and preventing lipid peroxidation by lowering liver lipid profiles and lipoperoxide products [25].

This study aims to investigate whether a high-fat diet during pregnancy could cause AFLP in both mother and fetus. The protective property of Spirulina against AFLP in mother was also investigated. Although it has been proofed that Spirulina has a protective effect on the liver, there is no report on the effect of Spirulina on the fetal liver during pregnancy. Therefore, the objective of this research is to evaluate the prevention effect of Spirulina on the fetal liver of mice fed with a high-fat diet during pregnancy and study this organ histomorphometrically.

\section{METHODS}

\section{Preparing Spirulina pellets and fat emulsion}

Powdered Spirulina platensis used in the experimental diet was purchased from Riz Jolbak Parsian Co. The daily needed pellet of each mouse was ground into powder, and then Spirulina was added to it at the ratio of 1:10, and it was mixed with warm water so it would turn into a paste. Then, we turned the paste into pellets suitable for the animals to consume and kept them at room temperature until they dried.

The definition of high-fat-diet is different from humans to the mouse. Standard mouse diets contain lower amounts of fat than the recommended human diet. In contrast to approximately $30 \%$ of total energy intake from fats in humans, the standard mouse diets contain less than $10 \%$ of kcal fat, whereas high-fat diets and very high-fat diets contain $30 \%-50 \%$ and more than $50 \%$ of kcal fat, respectively [26,27]. In this project, the method proposed by Zou et al. [28] was used to induce steatosis in the experimental groups. The high-fat emulsion diet contained $77 \%$ of its energy from fat, $14 \%$ from total milk powder, and $9 \%$ from carbohydrates. The composition of macronutrients in this emulsion is shown in Table 1. In the prepared emulsion, proteins were provided by total milk powder, carbohydrates by saccharose, and fat by corn oil. Each diet was supplemented with a vitamin and mineral mixture. The emulsion was stored at $4^{\circ} \mathrm{C}$, heated in a $42^{\circ} \mathrm{C}$ water bath, and fully mixed before use.

\section{Experimental animals}

Inducing fatty liver

Sixty small female NMRI mice weighing $28 \pm 3$ g were supplied by the Department of Experimental Animals of Pasteur Institute. To acclimate them to the new environment, the mice were kept in special cages for 1 week before the beginning of the experiment on a 12-h light/dark cycle and temperature of $2 \pm 23^{\circ} \mathrm{C}$ with sufficient supply of water and
Table 1: Composition of the high-fat emulsion diet ingested through gavage to mice

\begin{tabular}{ll}
\hline Component & Dosage \\
\hline Corn oil & $400 \mathrm{~g}$ \\
Sucrose & $150 \mathrm{~g}$ \\
Whole milk powder & $80 \mathrm{~g}$ \\
Cholesterol & $100 \mathrm{~g}$ \\
Sodium deoxycholate & $10 \mathrm{~g}$ \\
Tween 80 & $36.4 \mathrm{~g}$ \\
Propylene glycol & $31.1 \mathrm{~g}$ \\
Multi vitamin & $2.5 \mathrm{~g}$ \\
Salt & $10 \mathrm{~g}$ \\
Mixed minerals & $1.5 \mathrm{~g}$ \\
Distilled water & $300 \mathrm{ml}$ \\
Total energy & $43-42$ \\
\hline
\end{tabular}

standard food, produced by Behparvar Co. Experimental procedures were approved by the Ethics Committees for Animal Experiments of Parand Azad University. The mice were randomly assigned to four groups as follows:

- Group (C): The healthy control group

- $\quad$ Group (C-): The group fed with high-fat diet (as the negative control)

- Group $(\mathrm{C}+$ ): The group fed with high-fat diet + Simvastatin (as the positive control)

- Group (SP): The group fed with high-fat diet + Spirulina

To get liver steatosis, the negative control group received $10 \mathrm{ml} / \mathrm{kg}$ body weight of lipid emulsion every day for 56 days through gavage. Simultaneously, the positive control group received a daily dose of a suspension of $75 \mathrm{mg} / \mathrm{kg}$ body weight Simvastatin in $0.1 \%$ methylcellulose through gavage [29]. The Simvastatin was given through gavage to the group $(\mathrm{C}+)$ to protect the liver against steatosis, and they were examined for histopathological changes of the liver and the results compared with the Group (SP). The SP group received Spirulina in their diet in addition to gavage of fat emulsion, and finally, $10 \mathrm{ml} / \mathrm{kg}$ body weight of normal saline was given through gavage to the mice in the control group.

\section{Pregnancy}

The mice become pregnant after 56 days ( 8 weeks) of diets through polygamy. The two female mice were mated with one male mouse. Dams failing to become pregnant were allowed to remate. Day zero of pregnancy was determined by the formation of a vaginal plug. Dams were maintained on their diets through pregnancy. On the $17^{\text {th }}$ day, the mice were anesthetized using ketamine and xylazine, and the cesarean section was performed and the fetuses and placenta were removed from uterine horns. Five fetuses were selected randomly from each pregnant mouse, and they were dissected using stereomicroscope and their livers were removed for pathological examinations.

\section{Morphological evaluation}

For histopathological analysis, liver specimens fixed in $10 \%$ neutralbuffered formalin for $24 \mathrm{~h}$ and were then embedded in paraffin, sliced at $5-\mu \mathrm{m}$ thickness and stained with hematoxylin and eosin (H\&E) staining for detection the degree of hepatic steatosis [30]. The pathological changes were assessed and photographed under a Nikon optical microscope (ECLIPSE E200). Liver biopsy for liver steatosis based on lipid changes in hepatocytes was scored according to Takahashi and Fukusato [31] as follow zero: No steatosis, normal liver; I: $<25 \%$ of hepatocytes affected; II: $26-50 \%$ of hepatocytes affected; III: $51-75 \%$ of hepatocytes affected; and IV: More than $76 \%$ of hepatocytes affected. The cells were counted using a lens with a rectangular framework measuring $83 \mu \mathrm{m} \times 62 \mu \mathrm{m}$ on an area of $5146 \mu \mathrm{m}^{2}$. Grading was done at $\times 40$ in ten microscopic fields.

\section{Total bilirubin measurement}

To test the changes in total bilirubin, which is one of the symptoms of AFLP, blood samples were taken from the heart before and during 
pregnancy. The serum total bilirubin was assayed according to Gaber [32].

\section{Statistical analysis}

SPSS statistical software was used to analyze the data. Quantitative data were expressed as mean \pm standard deviation, and Scheffe post hoc test was used to examine differences between groups and one-way ANOVA was used to compare liver fat levels across all groups. The significance level was set at $\mathrm{p}<0.05$

\section{RESULTS}

\section{The effect of Spirulina platensis on maternal blood bilirubin} The effect of Spirulina platensis on maternal blood bilirubin in mothers with AFLP before and during pregnancy is shown in Table 2. According to Table 2, before pregnancy, serum bilirubin levels increased significantly in the negative group compared with all other groups. Serum bilirubin levels increased significantly in the Group (C+) and (SP) compared with the control group. However, it decreased compared with the Group (C-). Compared with Group (SP), serum bilirubin levels decreased significantly in the Group $(\mathrm{C}+)(\mathrm{p}<0.05)$. The results also showed that serum bilirubin levels during pregnancy increased significantly in the Group (C-) compared with the other groups. Serum bilirubin level increased significantly in the Group $(\mathrm{C}+)$ and $(\mathrm{SP})$ compared with the Group (C); however, compared with the Group (C+), it decreased significantly in the Group (SP) $(\mathrm{p}<0.05)$.

\section{Histopathology of the effect of Spirulina platensis on fetal liver damage}

In microscopic examinations, there was no abnormal condition such as necrosis and infiltration of inflammatory cells in the fetal livers tissue of the control healthy group (Fig. 1a); however, in the liver of fetuses whom mothers fed with a high-fat diet for 8 weeks, Group (C-), lipid changes, and fatty liver in mothers caused heavy damages to the

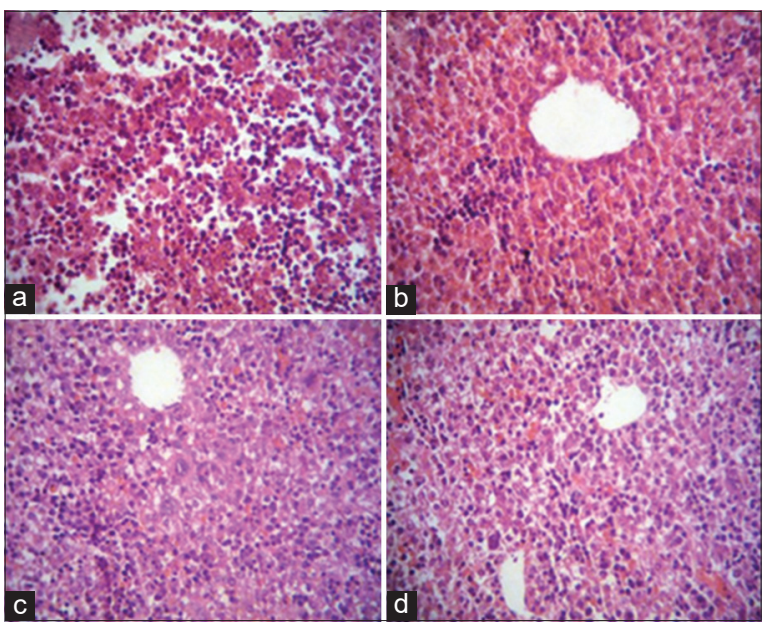

Fig. 1: Histological changes in the liver of 17-day fetuses from dams with the following diets: (a) Group (C), (b) Group (C-), (c) Group (C+), and (d) Group (SP), (Staining by haematoxylineosin and magnification of $\times 40$ )

Table 2: Bilirubin level (TB) before pregnancy and during pregnancy

\begin{tabular}{lll}
\hline Treatment & $\begin{array}{l}\text { TB }(\mathbf{m g} / \mathbf{d l}) \text { before } \\
\text { pregnancy }\end{array}$ & $\begin{array}{l}\text { TB }(\mathbf{m g} / \mathbf{d l}) \text { during } \\
\text { pregnancy }\end{array}$ \\
\hline Group (C) & $0.33 \pm 0.01$ & $0.39 \pm 0.02$ \\
Group (C-) & $0.90 \pm 0.04$ & $0.85 \pm 0.04$ \\
Group (C+) & $0.35 \pm 0.04$ & $0.44 \pm 0.03$ \\
Group (SP) & $0.31 \pm 0.04$ & $0.58 \pm 0.03$ \\
\hline
\end{tabular}

Data expressed as mean \pm standard deviation for each group $(\mathrm{p}<0.005)$ fetal livers as well, and a big part of hepatocytes was destroyed, and hyperemia and infiltration of inflammatory cells into hepatocytes can be seen. In addition, sinusoidal spaces are tighter and the concentration of blood islands is high (Fig. 1b). In the Group (C+), which had received a high-fat diet plus Simvastatin, more inflammation and necrosis were seen than those in the Group (SP). In fact, in Group (C+) hyperemia and infiltration of inflammatory cells accompanied liver damage and necrosis of hepatocytes. In comparison, Group ( $\mathrm{C}+$ ) shows more hepatocyte concentrations, wider sinusoidal spaces, and fewer blood islands than Group (C-) (Fig. 1c). In the fetal livers of the Group (SP), hepatic hyperemia and inflammatory cells were seen but liver damage and concentration of inflammatory cells were less than those in the Group $(\mathrm{C}+)$. In addition, compared with the negative control group, there is a high concentration of hepatocytes, small narrow sinusoidal spaces, and few blood islands, signifying the effectiveness of Spirulina (Fig. 1d).

\section{Cell count}

To examine the disease more closely, the liver fat content of the fetus livers was measured, and hepatocytes, macrophages, blood islands, bile ducts, and megakaryocytes were counted and results are furnished in Table 3. The results showed that fetus liver fat content in Group Cincreased significantly compared with the Group $\mathrm{C}$ while the fat content of the fetus from the Group SP is in the range of the fat content of the Group C+. Moreover, no histomorphometric change was seen in the liver tissue of the fetuses in the Group C. Hepatocyte changes in the fetal livers in Spirulina and positive groups decreased compared with the control group, but they increased compared with the negative group. There was no significant difference in the macrophage variable between the control and Spirulina groups; however, it decreased significantly in the control group compared with the positive and negative groups $(\mathrm{p}<0.001)$. In addition, it decreased significantly in the positive group compared with the negative group. The blood islands variable increased significantly in the negative group compared with the other groups, and it increased significantly in Spirulina and positive groups compared with the control group, but it decreased significantly compared with the negative group. The bile duct variable increased significantly in Spirulina and positive groups compared with the control group, but it decreased significantly compared with the negative group. The megakaryocytes variable increased significantly in the negative group compared with the positive and control groups, but it was not significantly different from the Spirulina group, and it decreased significantly in the positive group compared with the other groups. The liver fat was significantly lower in the control group compared with the Spirulina and negative groups. In addition, although the liver fat was lower in the control group than in the positive group, the difference was not significant. Finally, the liver fat in the Spirulina and positive groups was significantly lower than that in the negative group.

Table 4 shows the result of one-way ANOVA of the liver fat scores and cell variables in the liver of the fetus in the four groups of $\mathrm{C}, \mathrm{C}-, \mathrm{C}+$, and SP. A significant difference across all four groups in the liver fat $(p<0.001)$, hepatocytes $(p<0.001)$, and macrophages $(p<0.001)$ can be observed. In addition, no significant difference was seen across the four groups in blood islands $(p=0.615)$, bile ducts $(p=0.611)$, and megakaryocytes $(\mathrm{p}=0.973)$. Scheffe post hoc test was used to examine the differences in liver fat, the result of which is shown in Table 5.

As shown in Table 5, liver fat is significantly lower in the control group than in Spirulina $(\mathrm{p}<0.003)$ and negative $(\mathrm{p}<0.001)$ groups. In addition, although there was a difference in liver fat between the control group and the positive group, the difference was not statistically significant $(p=0.168)$. The liver fat in the Spirulina group was more than that in the positive group, but the difference was not significant $(p=0.424)$. On the other hand, liver fat in the Spirulina group was significantly lower than that in the negative group $(\mathrm{P}=0.001)$. Finally, liver fat in the positive group was significantly lower than that in the negative group $(\mathrm{p} \leq 0.001)$. 
Table 3: Mean and standard deviation of liver fat content and cell variables in the liver in the four groups of C, C-, C+, and SP

\begin{tabular}{llll}
\hline Variable & Control & Spirulina & Positive \\
\hline Liver fat & $1.60 \pm 0.70$ & $2.90 \pm 0.56$ & $2.30 \pm 0.48$ \\
Hepatocytes & $106.8 \pm 10.51$ & $101.50 \pm 24.71$ & $84.90 \pm 20.04$ \\
Macrophages & $2.60 \pm 1.07$ & $2.60 \pm 0.84$ & $6.0 \pm 1.76$ \\
Blood islands & $0.90 \pm 0.73$ & $1.20 \pm 1.03$ & $1.30 \pm 1.15$ \\
Bile ducts & $0.30 \pm 0.48$ & $0.40 \pm 0.51$ & $0.40 \pm 0.51$ \\
Megakaryocytes & $2.10 \pm 0.73$ & $2.20 \pm 1.39$ & $2.70 \pm 0.78$ \\
\hline
\end{tabular}

Table 4: One-way ANOVA for comparing the liver fat scores and cell variables in the fetus liver in the four groups of (C), (C-), (C+), and (SP)

\begin{tabular}{lll}
\hline Variable & $\mathbf{F}$ & $\mathbf{p}$ \\
\hline Liver fat & 27.72 & $\mathrm{p}<0.001$ \\
Hepatocytes & 8.28 & $\mathrm{p}<0.001$ \\
Macrophages & 44.35 & $\mathrm{p}<0.001$ \\
Blood islands & 0.606 & 0.615 \\
Bile ducts & 0.613 & 0.611 \\
Megakaryocytes & 0.075 & 0.973 \\
\hline
\end{tabular}

Table 5: Scheffe post hoc test to examine pairwise differences in liver fat

\begin{tabular}{lllll}
\hline Variable & Group & Group & $\begin{array}{l}\text { Mean } \\
\text { differences }\end{array}$ & p \\
\hline Liver fat & Control & Spirulina & -1.20 & 0.003 \\
& & Positive control & -0.70 & 0.168 \\
& & Negative control & -2.60 & $<0.001$ \\
& Spirulina & Positive control & 0.50 & 0.424 \\
& & Negative control & -1.40 & 0.001 \\
& Positive & Negative control & -1.90 & $<0.001$ \\
& control & & & \\
\hline
\end{tabular}

\section{DISCUSSION}

This study was aimed at investigating the protective effect of Spirulina platensis on the fetal liver of mice with AFLP. The results showed that there was a significant difference in maternal total serum bilirubin between the experimental groups and the control group before and during pregnancy. In fact, quantification of serum bilirubin is evidence for the assessment of liver function and an unusual increase in the levels of bilirubin in the serum indicates severe perturbation of hepatocellular function. In this research, the bilirubin level in the Spirulina group before pregnancy increased significantly compared with the positive and control groups, but it decreased significantly compared with the negative group $(\mathrm{p}<0.05)$. Then, after pregnancy, the bilirubin level in the Spirulina group showed a significant decrease compared with all the other three groups $(\mathrm{p}<0.05)$. This finding shows that Spirulina could probably treat bile duct damage in the liver (Table 3 ) and could be able to strengthen biliary dysfunction. Similar protective effects were observed for the Spirulina fusiformis [20]. A lot of studies have investigated the effect of Spirulina on different blood parameters and even on liver enzymes, all of which have pointed out the beneficial effects of this cyanobacterium. Therefore, we can consider Spirulina as an effective dietary supplement in reducing enzyme disorders, bile duct disorders, and other complications. Studies have shown that this alga is a probiotic organism that enhances health [33]. This alga is rich in unsaturated fatty acids, phycocyanin [34], and phenolic compounds [35]. Different researchers have studied the effect of Spirulina on proteins and blood lipids [36,37], anti-oxidant properties especially phycocyanin, its antiviral, and immunomodulatory properties in animals [38]. Studies have found that oxidative stress is involved in many liver diseases, and it leads to liver damage $[20,39]$. Therefore, antioxidants play an important role in scavenging free radicals and thereby protecting the liver. In addition, Spirulina contains plenty of superoxide dismutase
(SOD) (an antioxidant enzyme), which removes free radicals [40]. Many algae and cyanobacteria are rich in phycocyanobilin (PCB) and are considered rich sources of the chromophore, which helps absorb light as a component of holoprotein phycocyanin [41]. PCB is one of the derivatives of biliverdin that, in mammals, is expressed by biliverdin reductase to phycocyanobilin, which is quite similar to bilirubin in structure. Studies have shown that about $0.66 \%$ of the dry mass of Spirulina is PCB (phycocyanin comprises around 14\% of the total dry weight of Spirulina and PCB constitutes $4.7 \%$ of the mass of phycocyanin), making it easy to be absorbed by the body and to have a high antioxidant activity (15-29). Considering the findings of studies on the effect of Spirulina on blood factors, we could probably conclude that the existence of the abovementioned compounds in this alga reduced the bilirubin level during pregnancy in Spirulina group compared with the other groups in the present study. However, studies show that this is a pathologic condition and requires more examinations. In general, the serum bilirubin level is an indicator of the liver function and abnormal increase in bilirubin level indicates a severe disorder of hepatocellular function [42]. Unlike HELLP syndrome and liver complication in pre-eclampsia and eclampsia, AFLP is a disease with real liver dysfunction. In AFLP, hyperbilirubinemia is seen without hemolysis; however, in pre-eclampsia, hyperbilirubinemia is rare and is in interaction with hemolysis [4]. In severe cases of pre-eclampsia, elevated bilirubin level to less than $6 \mathrm{mg} / \mathrm{dL}$ has been reported [2]. On the other hand, elevated bilirubin level (usually above $5 \mathrm{mg} / \mathrm{dc}$ ) indicates AFLP [43]. The findings of the present study about bilirubin levels support the findings of studies by other researchers $[4,10,43]$. Studies have also found that bilirubin or its precursor (biliverdin) could prevent a large range of disorders as an oral antioxidant. Recent studies have found that bilirubin in nanomolar concentrations acts as a powerful inhibitor of nicotinamide adenine dinucleotide phosphate (NADPH) oxidase. Expression of heme oxygenase- 1 is induced by intracellular oxidant stress, which produces bilirubin from heme through biliverdin; this mechanism provides feedback control of the oxidant stress-mediated by NADPH oxidase. Bilirubin's suppressive impact on NADPH oxidase activity likely explains the growing epidemiological literature that associates increased serum bilirubin, or high-expression polymorphisms of heme oxygenase-1, with diminished risk for vascular disorders, certain cancers, and various other diseases. Recent molecular studies have pointed out that the cause of AFLP is probably mitochondrial dysfunction. Some studies have shown that AFLP is like autosomal diseases that result from fatty acids oxidation defects. The least likely cause of AFLP is beta-oxidation defect. The pathogenesis of AFLP still seems to be elusive. A lot of studies have pointed out that this disease is directly and indirectly associated with the deficiency of the long-chain 3-hydroxyacyl-CoA dehydrogenase in the fetus, which is a fatty acid beta-oxidation defect [9]. The fetal liver tissue was investigated in mice with AFLP in the present study.

At the beginning of the study, examining the liver tissue showed that lipid affected liver structure and caused liver steatosis. Then, semi-thin sections of the livers were prepared to be investigated by a microscope. Histological studies showed that the increase in the intake of fat by the mother affected both maternal and fetal liver. These examinations also showed that due to the high intake of fat, lipid vesicles increased in all experimental groups (negative, Spirulina, and positive) compared with the control group, which showed deposition of lipid in fetal liver cells. It was also showed that in the Spirulina group, lipid vesicles, hepatic 
hyperemia, and inflammatory cells were seen, but they decreased significantly compared with the positive and negative groups and increased significantly compared with the control group (Fig. 1d) $(p<0.05)$. In other words, Spirulina had a positive effect on both maternal and fetal livers. Considering the findings of the present study, we can say that Spirulina decreases deficiencies in lipid metabolism resulting from a high-fat diet. In addition, the findings showed that in Spirulina and positive groups, forming lipid vesicles in liver parenchymatous cells decreased significantly compared with the negative group, signifying the effectiveness of Spirulina and Simvastatin in decreasing lipid deposition in liver structure (Fig. 1c and d). This finding is supported by the findings of the study by Blé-Castillo et al. [29]. In fact, the findings showed that high-fat diet affects lipid metabolism in fetal liver, and the existence of lipid vesicles in fetal livers is a sign of lipid concentration and increased lipid deposition $[44,45]$ stated that factors such as hepatocyte dysfunction (especially mitochondria), deficiency in prolactin proliferator receptors, and receptor inflammation are involved in causing NASH. In a similar study, Pruis et al. [44] stated that regardless of electron transport, signs of change in mitochondrial function, microvesicular fat accumulation, decreased plasma b-hydroxybutyrate, and downregulation of PPARA mRNA are seen. We can assume that peroxisomal and mitochondrial b-oxidation systems are not properly regulated so that they can adapt to the increase in fatty acids production. The hepatic triglyceride accumulation prevents regulation of fatty acid synthesis, mitochondrial B-oxidation, and fatty acid export. These factors could play an important role in causing AFLP in both mother and fetus.

The liver tissue was then examined and it was observed that the number of hepatocytes increased significantly in the Spirulina group compared with the positive and negative groups; however, it decreased significantly compared with the control group $(p<0.001)$. This finding shows that Spirulina could prevent hepatocyte apoptosis. Examining hepatocytes showed a significant increase in the number of hepatocytes in the control group compared with the other groups. In fact, this finding shows that hepatocyte apoptosis is an important pathologic feature of NAFLD $[46,47]$. In addition, hepatocyte apoptosis and inflammation have been seen as directly associated with fibrosis. Different studies on NAFLD in humans and animals and during in vitro hepatocyte steatosis have shown that the accumulation of lipid vesicles in liver cells increases apoptosis [47]. This fact shows an association between hepatocyte apoptosis and liver fibrogenesis [48]. Fibrogenic activity is started by engulfment of apoptotic bodies by Ito cells in these cells, and it may be how hepatocyte apoptosis promotes fibrosis [49]. In the present study, we showed that hepatocyte apoptosis predicts NASH accurately [46]. Then, further examinations showed a significant increase in the number of macrophages in this tissue in the negative and positive groups compared with Spirulina and control groups $(p<0.001)$. We know that chronic inflammation and macrophage infiltration into adipose tissue are found in AFLP [50]. In this study, in addition to changes in sinusoidal blood, considerable changes were seen in the phagocytic activity of liver macrophages. Similar results were also reported by McCurdy et al. [51] and Frazier et al. [52]. As macrophages release reactive oxygen species, nitro radicals, cytokines, and vasoactive prostanoids when they are in the activated phase, they may cause parenchymal injury, the microvascular inflammatory response, and activation of the neighboring Ito cells.

Blood islands decreased significantly in the control group compared with the other groups; in addition, they decreased significantly in the Spirulina group compared with the positive and negative groups $(p=0.1)$. Bile ducts in the control and Spirulina groups decreased significantly compared with the positive and negative groups, and compared with the control group, it increased significantly in the Spirulina group $(\mathrm{p}=0.1)$.

Histological studies of the experimental groups (positive, negative, and Spirulina) are showed widespread changes in sinusoidal spaces. The sinusoidal spaces were dilated in the control group compared with the other groups (Fig. 1a), but in the other groups, these spaces were reduced depending on the type of treatment (Fig. 1b-d). Studies have shown that enlarged hepatic parenchymal cells, which are swollen with lipid, cause reductions in sinusoidal perfusion [53].

These structural changes in sinusoids were seen in the negative group (Fig. 1b), and they make them inefficient conduits, which finally causes damage to liver tissue [54]. Like the present study, other studies have also found considerable reductions in the number of sinusoids [54-57]. These alterations are accompanied by progressive damages to the sinusoidal endothelial cells (SEC), as well as the development of a basal lamina and deposition of collagen in Disse space [54]. In addition, adhesion of leukocytes to the sinusoidal endothelium may exist, followed by leukocyte infiltration into the hepatic parenchyma to form inflammatory foci [58]. On the other hand, microvascular blood flow is further restricted due to the deposition of collagen in Disse space as well as the narrowing and distortion of the sinusoidal lumen. This situation gets worse when leukocytes are trapped in the narrowed sinusoids or adhere to the endothelium following a hepatic microvascular inflammatory response. Furthermore, lipid accumulation in hepatic parenchymal cells and oxidative stress seem to be more important factors than the microvascular changes in steatosis. Studies have shown that interrupted sinusoidal blood flow because of lipid accumulation in parenchymal cells and collagen deposition in Disse space seems to be linked with hepatocellular injury in AFLP [53].

One of the most important findings of the present study was the fact that a persistent high-fat diet caused more lipid transfer to the fetus. Lipolysis from maternal adipose tissue increases plasma glycerol concentration and fatty acids [51]. This situation along with dietary fatty acids and hepatic triglycerides (TGs) results in an increase in TG-rich lipoproteins in the mother's circulation and consequently increases the transfer of fatty acids to the fetus [51,59-61]. Lipotoxicity is a major concern when lipid is accumulated within the liver. It could result in insulin resistance, oxidative stress, activation of pro-inflammatory cytokines, and liver fibrosis in the end [51,62-65]. The obtained results show that the chance of lipid accumulation in the fetal liver soars when there is exposure to maternal lipid-derived fuels during early pregnancy. This lipid accumulation is linked with lipotoxicity, which may result in macrophage infiltration and elevated production of inflammatory cytokine. The oxidative stress pathway is activated due to this inflammation, and it can activate the transcriptional regulators of hepatic gluconeogenesis [51]

In summary, the findings of the present study show that Spirulina has a more beneficial effect on the fetal liver than Simvastatin as a chemical medicine. Spirulina proved effective in minimizing highfat-diet-induced damage to lipid metabolism. It consists of protein (55\%-70\%), carbohydrates (15\%-25\%), minerals, essential fatty acids (18\%), vitamins especially B and E, high levels of beta carotene, and zeaxanthin, pigments such as phycocyanin, carotenoid, Chlorophyll, and other substances. In addition, Spirulina has high levels of Gammalinolenic acid and it can have a protective effect on liver against toxins as the main causes of free radicals, cirrhosis, steatosis, and necrosis in liver cells [40]. In general, the body is endowed with systems of enzymatic and non-enzymatic antioxidants that scavenge and neutralize free radicals and prevent damage to other tissues. The antioxidant systems include SOD, glutathione peroxidase, catalase, and macromolecules such as albumin, ferritin, ceruloplasmin, and also molecules such as uric acid, bilirubin, ascorbic acid, and alpha-tocopherol [66,67]. Studies have shown that antioxidants contain elements that control and scavenge free radicals produced during the natural metabolism of the body. Blé-Castillo et al. [29] observed the protective effect of Spirulina against free radicals as well as oxidative stress, toxins, and medications due to the presence of antioxidants, tocopherols, phenolic acids, phycocyanin, selenium, beta-carotene, and flavonoid compounds. We know that scavenging free radicals are one of the main mechanisms that prevent free radical chain reactions. Studies have shown that the liver and kidney are the first targets of free radicals as they are exposed 
to toxic and xenobiotic substances more the other tissues [68]. Having antioxidant properties and being rich in phycocyanin C, Spirulina could reduce free radicals and consequently protect the maternal and fetal liver. In addition, phycocyanin C plays a key role in scavenging lipid and peroxidation chain reactions. SODs, members of the metalloenzymes family, have also been known as antioxidants that scavenge free radicals. In fact, when free radicals increase in the body and the existing antioxidants do not suffice to combat them, phycocyanin in Spirulina regulates the enzymatic activity and restores the body's capability to fight free radicals. In addition to phycocyanin $\mathrm{C}$, beta-carotene and tocopherols are other antioxidants found in Spirulina [38]. In additionally, Spirulina has Vitamin E, which is an antioxidant against lipid peroxidation chain. Vitamin E is a lipid-soluble antioxidant that prevents the peroxidation of unsaturated lipids [67]. Studies on the protective effect of Vitamin E suggest that this vitamin removes free radicals from hydrophobic environments $[42,69]$. In fact, the radical $(\mathrm{R})$ is deactivated by a molecule of Vitamin $\mathrm{E}$ ( $\alpha$-Tocopherol) $[69,70]$. It seems after absorption of in vivo Vitamin $\mathrm{E}$, it reacts with free radicals and probably with oxidizing intermediates. Therefore, Vitamin E prevents free radical chain reaction and protects cell membranes [69,71]. Finally, the therapeutic effect of Spirulina in treating liver diseases found in this study is supported by the findings of other studies done on Spirulina.

\section{CONCLUSIONS}

The findings of the present study showed that Spirulina has a protective and beneficial effect on the fetal liver in AFLP in mice. The protective efficiency of Spirulina is very promising as evidenced by histopathological studies. The AFLP protective property of the Spirulina is attributed to the presence of various constitutions which are present in Spirulina. Some other medications used for the treatment of fatty liver could probably be used during pregnancy too; however, some of them are dangerous for the fetus and cannot be used during pregnancy. However, such an effect did not observe in the case of Spirulina. As maternal nutrition is important during pregnancy, proper nutrition could ensure the health and development of the fetus; however, incorrect or unbalanced maternal nutrition causes fetal disorders. In addition, it is well-known that the liver detoxifies the body, and during the prenatal period, all substances that pass through the placenta enter the fetal liver directly. If these substances are toxic, they affect liver development and function adversely. Therefore, considering the findings of the present study, Spirulina having all the aforementioned nutrition could be considered as a green, non-toxic, and beneficial dietary supplement during pregnancy and reduce the risk of fetal disorders. It is worth noting that this study has been done on animals and whether it has similar effects on humans requires further studies.

\section{ACKNOWLEDGMENT}

The authors would like to thank the Central Tehran Branch Azad University and Riz Jolbak Parsian Company for their support on conducting this research with the research study identification number 10130517942002.

\section{AUTHORS' CONTRIBUTIONS}

The author declares that all the named authors have contributed equally to this article.

\section{CONFLICTS OF INTEREST}

The authors have no conflicts of interest to disclose.

\section{FUNDING}

This research did not receive any specific grant from funding agencies in the public, commercial, or non-for-profit sectors.

\section{REFERENCES}

1. Shaker M, Tabbaa A, Albeldawi M, Alkhouri N. Liver transplantation for nonalcoholic fatty liver disease: New challenges and new opportunities. World J Gastroenterol 2014;20:5320-30.

2. Sherlock S, Dooley J. The liver in pregnancy. In: Sherlock S, Dooley J, editors. Diseases of the Liver and Biliary System. London: Blackwell Science; 2002. p. 472-5.

3. Lee NM, Brady CW. Liver disease in pregnancy. World J Gastroenterol 2009; $15: 897$.

4. Gargari SS. Acute fatty liver in pregnancy with complications of scites and bilateral pleural effusion in pregnant woman. Sci J Kurdistan Uni Medic Sci 2007;12:84-90.

5. Burroughs AK, Seong NG, Dojcinov DM, Scheuer PJ, Sherlock SV. Idiopathic acute fatty liver of pregnancy in 12 patients. QJM Int J Med 1982:51:481-97.

6. Chalifoux MR, Blank HL. Acute fatty liver of pregnancy and disseminated intravascular coagulation: A case report. J Obstet Anaesth Criti Care 2020;10:42

7. Browning MF, Levy HL, Wilkins-Haug LE, Larson C, Shih VE. Fetal fatty acid oxidation defects and maternal liver disease in pregnancy. Obstet Gynecol 2006;107:115-20.

8. Treem W. Mitochondrial fatty acid oxidation and acute fatty liver of pregnancy. Semin Gastrointest Dis 2002;13:55-66.

9. Ibdah JA. Acute fatty liver of pregnancy: An update on pathogenesis and clinical implications. World J Gastroenterol 2006;12:7397.

10. Natarajan SK, Thangaraj KR, Eapen CE, Ramachandran A, Mukhopadhya A, Mathai M, et al. Liver injury in acute fatty liver of pregnancy: Possible link to placental mitochondrial dysfunction and oxidative stress. Hepatology 2010;51:191-200.

11. Mannaerts GP, Debeer LJ, Thomas J, De Schepper PJ. Mitochondrial and peroxisomal fatty acid oxidation in liver homogenates and isolated hepatocytes from control and clofibrate-treated rats. J Biol Chem 1979;254:4585-95

12. Al-Ani OA. Drugs in pregnancy. Asian J Pharm Clin Res 2020;13:78-82.

13. Hoseini SM, Khosravi-Darani K, Mozafari MR. Nutritional and medical applications of spirulina microalgae. Mini Rev Med Chem 2013;13:1231-7.

14. Ku CS, Yang Y, Park Y, Lee J. Health benefits of blue-green algae: Prevention of cardiovascular disease and nonalcoholic fatty liver disease. J Med Food 2013;16:103-11.

15. Ramzi GA, Puneeth HR, Madhu CS, Sharada AC. Antagonistic effects of combination of flaxseed oil and spirulina platensis oil on their biological properties. Int J Pharm Pharm Sci 2015;7:122-7.

16. Serban MC, Sahebkar A, Dragan S, Stoichescu-Hogea G, Ursoniu S, Andrica F, et al. A systematic review and meta-analysis of the impact of spirulina supplementation on plasma lipid concentrations. Clin Nutr 2016;35:842-51.

17. Maniyar R, Umashankar GK. Effectiveness of spirulina mouthwash on reduction of dental plaque and gingivitis: A clinical study. Int J Pharm Pharm Sci 2017;9:136-9.

18. Ferreira-Hermosillo A, Torres-Duran PV, Juarez-Oandropeza MA. Hepatoprotective effects of spirulina maxima in patients with nonalcoholic fatty liver disease: A case series. J Med Case Rep 2010;4:103.

19. Deng R, Chow TJ. Hypolipidemic, antioxidant, and antiinflammatory activities of microalgae spirulina. Cardiovasc Ther 2010;28:e33-45.

20. Vedi M, Kalaiselvan S, Rasool M, Sabina EP. Protective effects of blue green algae spirulina Fusiformis against galactosamine-induced hepatotoxicity in mice. Asian J Pharm Clin Res 2013;6:150-4.

21. Coskun ZK, Kerem M, Gurbuz N, Omeroglu S, Pasaoglu H, Demirtas C, et al. The study of biochemical and histopathological effects of spirulina in rats with TNBS-induced colitis. Br Med J 2011;112:235-43.

22. Ismail MF, Ali DA, Fernando A, Abdraboh ME, Gaur RL, Ibrahim WM, et al. Chemoprevention of rat liver toxicity and carcinogenesis by spirulina. Int J Biol Sci 2009;5:377

23. Khan M, Shobha JC, Mohan IK, Naidu MU, Sundaram C, Singh S, et al. Protective effect of spirulina against doxorubicin-induced cardiotoxicity. Phytother Res 2005;19:1030-7.

24. Karkos PD, Leong SC, Karkos CD, Sivaji N, Assimakopoulos DA. Spirulina in clinical practice: Evidence-based human applications. Evid Based Complement Altern Med 2011;2011:531053.

25. El-Baky HH, El Baz FK, El-Baroty GS. Enhancement of antioxidant production in spirulina Platensis under oxidative stress. Acta Physiol Plant 2009;31:623

26. Kucera O, Cervinkova Z. Experimental models of non-alcoholic fatty liver disease in rats. World J Gastroenterol 2014;20:8364-76.

27. Fellmann L, Nascimento AR, Tibiriça E, Bousquet P. Murine models for pharmacological studies of the metabolic syndrome. Pharmacol Ther 2013;137:331-40.

28. Zou Y, Li J, Lu C, Wang J, Ge J, Huang Y, et al. High-fat emulsioninduced rat model of nonalcoholic steatohepatitis. Life Sci 
2006;79:1100-7.

29. Blé-Castillo JL, Rodrıguez-Hernández A, Miranda-Zamora R, JuárezOropeza MA, Diaz-Zagoya JC. Arthrospira maxima prevents the acute fatty liver induced by the administration of simvastatin, ethanol and a hypercholesterolemic diet to mice. Life Sci 2002;70:2665-73.

30. Wang JQ, Li J, Zou YH, Cheng WM, Lu C, Zhang L, et al. Preventive effects of total flavonoids of Litsea coreana leve on hepatic steatosis in rats fed with high fat diet. J Ethnopharmacol 2009;121:54-60.

31. Takahashi Y, Fukusato T. Histopathology of nonalcoholic fatty liver disease/nonalcoholic steatohepatitis. World J Gastroenterol 2014;20:15539.

32. Garber CC. Jendrassik-grof analysis for total and direct bilirubin in serum with a centrifugal analyzer. Clin Chem 1981;27:1410-6.

33. Seyidoğlu N, Galip N. Effects of Saccharomyces cerevisiae and spirulina Platensis on growth performances and biochemical parameters in rabbits. Kafkas Univ Vet Fak Derg 2014;20:331-6.

34. Estrada JP, Bescós PB, Del Fresno AV. Antioxidant activity of different fractions of spirulina Platensis protean extract. Farmaco 2001;56:497-500.

35. Wu Q, Liu L, Miron A, Klímová B, Wan D, Kuča K. The antioxidant, immunomodulatory, and anti-inflammatory activities of Spirulina: An overview. Arch Toxicol 2016;90:1817-40

36. Nagaoka S, Shimizu K, Kaneko H, Shibayama F, Morikawa K, Kanamaru Y, et al. A novel protein C-phycocyanin plays a crucial role in the hypocholesterolemic action of spirulina Platensis concentrate in rats. J Nutr 2005;135:2425-30.

37. Riss J, Décordé K, Sutra T, Delage M, Baccou JC, Jouy N, et al. Phycobiliprotein C-phycocyanin from spirulina Platensis is powerfully responsible for reducing oxidative stress and NADPH oxidase expression induced by an atherogenic diet in hamsters. J Agric Food Chem 2007;55:7962-7

38. Finamore A, Palmery M, Bensehaila S, Peluso I. Antioxidant, immunomodulating, and microbial-modulating activities of the sustainable and ecofriendly spirulina. Oxid Med Cell Longev 2017;2017:3247528.

39. Valko M, Leibfritz D, Moncol J, Cronin MT, Mazur M, Telser J. Free radicals and antioxidants in normal physiological functions and human disease. Int J Biochem Cell Biol 2007;39:44-84.

40. Desai K, Sivakami S. Spirulina: The wonder food of the $21^{\text {st }}$ Century. Asia Pac Biotech News 2004;8:1298-302.

41. Brown SB, Houghton JD, Vernon DI. New trends in photobiology biosynthesis of phycobilins. Formation of the chromophore of phytochrome, phycocyanin and phycoerythrin. J Photochem Photobiol B 1990;5:3-23.

42. Martin A, Zulueta J, Hassoun P, Blumberg JB, Meydani M. Effect of Vitamin $\mathrm{E}$ on hydrogen peroxide production by human vascular endothelial cells after hypoxia/reoxygenation. Free Radic Biol Med 1996;20:99-105

43. Ghaffarnejad M. Acute fatty liver of pregnancy and preeclampsia in a triplet gestation. Acta Med Iran 2007;45:161-4

44. Pruis MG, Lendvai A, Bloks VW, Zwier MV, Baller JF, De Bruin A, et al. Maternal western diet primes non-alcoholic fatty liver disease in adult mouse offspring. Acta Physiol 2014;210:215-27.

45. Zhan YT, An W. Roles of liver innate immune cells in nonalcoholic fatty liver disease. World J Gastroenterol 2010;16:4652.

46. Wieckowska A, Zein NN, Yerian LM, Lopez AR, McCullough AJ, Feldstein AE. In vivo assessment of liver cell apoptosis as a novel biomarker of disease severity in nonalcoholic fatty liver disease. Hepatology 2006;44:27-33.

47. Feldstein AE, Canbay A, Guicciardi ME, Higuchi H, Bronk SF, Gores GJ. Diet associated hepatic steatosis sensitizes to fas mediated liver injury in mice. J Hepatol 2003;39:978-83.

48. Canbay A, Friedman S, Gores GJ. Apoptosis: The nexus of liver injury and fibrosis. Hepatology 2004;39:273-8.

49. Canbay A, Taimr P, Torok N, Higuchi H, Friedman S, Gores GJ. Apoptotic body engulfment by a human stellate cell line is profibrogenic. Lab Invest 2003;83:655.

50. Rius B, López-Vicario C, González-Périz A, Morán-Salvador E,
García-Alonso V, Clària J, et al. Resolution of inflammation in obesityinduced liver disease. Front Immunol 2012;3:257.

51. McCurdy CE, Bishop JM, Williams SM, Grayson BE, Smith MS, Friedman JE, et al. Maternal high-fat diet triggers lipotoxicity in the fetal livers of nonhuman primates. J Clin Invest 2009;119:323-35.

52. Frazier TH, DiBaise JK, McClain CJ. Gut microbiota, intestinal permeability, obesity-induced inflammation, and liver injury. J PEN J Parenter Enteral Nutr 2011;35:14S-20.

53. Farrell GC, Teoh NC, McCuskey RS. Hepatic microcirculation in fatty liver disease. Anat Rec 2008;291:684-92.

54. McCuskey RS, Ito Y, Robertson GR, McCuskey MK, Perry M, Farrell GC. Hepatic microvascular dysfunction during evolution of dietary steatohepatitis in mice. Hepatology 2004;40:386-93.

55. Sato N, Eguchi $H$, Inoue A, Matsumura $T$, Kawano $S$, Kamada $T$. Hepatic microcirculation in Zucker fatty rats. In: Longmuir IS, editor. Oxygen Transport to Tissue VIII. Berlin, Germany: Springer; 1986. p. 477-83.

56. Hasegawa T, Ito Y, Wijeweera J, Liu J, Malle E, Farhood A, et al. Reduced inflammatory response and increased microcirculatory disturbances during hepatic ischemia-reperfusion injury in steatotic livers of ob/ob mice. Am J Physiol Gastrointest Liver Physiol 2007;292:G1385-95.

57. Teoh NC, Ito Y, Field J, Bethea NW, Amr D, McCuskey MK, et al. Diannexin, a novel annexin $\mathrm{V}$ homodimer, provides prolonged protection against hepatic ischemia-reperfusion injury in mice. Gastroenterology 2007;133:632-46.

58. McCuskey RS, Nishida J, Eguchi H, McDonnell D, Baker GL, Ekataksin W, et al. Role of endotoxin in the hepatic microvascular inflammatory response to ethanol. J Gastroenterol Hepatol 1995;10:S18-23.

59. Herrera E, Ortega-Senovilla H. Lipid metabolism during pregnancy and its implications for fetal growth. Curr pharm Biotechnol 2014;15:24-31.

60. Herrera E, Amusquivar E, Lopez-Soldado I, Ortega H. Maternal lipid metabolism and placental lipid transfer. Horm Res Paediatr 2006;65:59-64.

61. Haggarty P. Placental regulation of fatty acid delivery and its effect on fetal growth-a review. Placenta 2002;23:S28-38.

62. Leclercq IA. Pathogenesis of steatohepatitis: Insights from the study of animal models. Acta Gastroenterol Belg 2007;70:25-31.

63. Picardi A, D'Avola D, Gentilucci UV, Galati G, Fiori E, Spataro S, et al. Diabetes in chronic liver disease: From old concepts to new evidence. Diabetes Metab Res Rev 2006;22:274-83.

64. Schäffler A, Schölmerich J, Büchler C. Mechanisms of disease: Adipocytokines and visceral adipose tissue-emerging role in nonalcoholic fatty liver disease. Nat Clin Pract Gastroenterol Hepatol 2005;2:273

65. Medina J, Fernández-Salazar LI, García-Buey L, Moreno-Otero R. Approach to the pathogenesis and treatment of nonalcoholic steatohepatitis. Diabetes Care 2004;27:2057-66.

66. Aksoy L, Sözbilir NB. Effects of Matricaria chamomilla L. on lipid peroxidation, antioxidant enzyme systems, and key liver enzymes in CCl4-treated rats. Toxicol Environ Chem 2012;94:1780-8.

67. Balahoroğlu R, Dülger H, Hanefi Özbek H, Bayram İ, Şekeroğlu MR. Protective effects of antioxidants on the experimental liver and kidney toxicity in mice. Eur J Gen Med 2008;5:157-64.

68. Mahmoodzadeh Y, Mazani M, Rezagholizadeh L, Abbaspour A, Zabihi E, Pourmohammad P. Effect of Tanacetum parthenium extract on total antioxidant capacity of tissues damaged by carbon tetrachloride in rats. J Ardabil Univ Med Sci 2017;16:363-73.

69. Ariamanesh S, Faghihi M, Kadkhodaei M. The effect of reperfusion ischemia on level vitamin e in venous blood and kidney tissue in rat. Cell J (Yakhteh) 2001;3:39-44.

70. Kontush A, Finckh B, Karten B, Kohlschutter A, Beisiegel U. Antioxidant and prooxidant activity of alpha-tocopherol in human plasma and low density lipoprotein. J Lipid Res 1996;37:1436-48.

71. Yoshikawa T, Yasuda M, Ueda S, Naito Y, Tanigawa T, Oyamada H, et al. Vitamin $\mathrm{E}$ in gastric mucosal injury induced by ischemiareperfusion. Am J Clin Nutr 1991;53:210S-4. 